\title{
Functional impact of endotoxin receptor CD14 polymorphisms on transcriptional activity
}

\author{
Jasmin Mertens • Rusudan Bregadze • \\ Ashham Mansur • Eva Askar • Heike Bickeböller • \\ Giuliano Ramadori • Sabine Mihm
}

Received: 23 February 2009/Revised: 17 April 2009/Accepted: 23 April 2009 /Published online: 27 May 2009

(C) The Author(s) 2009. This article is published with open access at Springerlink.com

\begin{abstract}
The polymorphism rs2569190 within the CD14 endotoxin (lipopolysaccharide, LPS) receptor gene is associated with various disease conditions that are assumed to rely on endotoxin sensitivity. In vitro experiments suggest that the $\mathrm{T}$ allele sensitizes the host for exogenous or endogenous LPS via an enhanced CD14 expression. To prove the impact of this single nucleotide polymorphism in its natural genomic context in vivo, two parameters of gene transcription were analyzed in peripheral blood mononuclear cells (PBMC) from single healthy individuals: (a) recruitment of RNA polymerase II by haplotype-specific chromatin immunoprecipitation and (b) the relative amount of transcripts by allele-specific transcript quantification (ASTQ). RNA polymerase II was found to be twice as much bound to the most prevalent haplotype, C-T-C-G, the only one carrying a $\mathrm{T}$ at the position rs2569190 of interest. ASTQ employing two independent read-out assays revealed, however, similar transcript numbers originating from C-T-C-G and non-C-T-C-G haplotypes. Total CD14
\end{abstract}

J. M. and R. B. contributed equally to this work.

J. Mertens $\cdot$ R. Bregadze $\cdot$ A. Mansur $\cdot$ E. Askar $\cdot$ G. Ramadori $\cdot$

S. Mihm

Department of Gastroenterology and Endocrinology,

University Medical Center Göttingen,

37075 Göttingen, Germany

H. Bickeböller

Department of Genetic Epidemiology, Georg-August-Universität,

37075 Göttingen, Germany

S. Mihm $(\bowtie)$

Department of Gastroenterology and Endocrinology,

University Medical Center Göttingen,

Robert-Koch-Straße 40,

37075 Göttingen, Germany

e-mail: smihm@med.uni-goettingen.de
mRNA levels from freshly isolated PBMC, moreover, were neither related to donors' geno- nor haplogenotypes. Our data argue for a functional impact of the rs 2569190 polymorphism in terms of a stronger transcription initiation on $\mathrm{T}$ allele gene variants even if preferential allele-specific binding does not result in an increase in transcript numbers. Endotoxin sensitivity associated with this genetic variation appears not to rely solely on a cis-acting regulatory impact of rs2569190 on CD14 gene transcription in PBMC.

Keywords SNP. LPS · Gene polymorphism ·

Gene expression · Innate immunity

\section{Introduction}

A host might get into contact with bacterially derived endotoxin (lipopolysaccharide, LPS) at the respiratory endothelium via environmental exposure of LPS containing dust, at the intestinal endothelium in the gut via the commensal microflora, at liver sinusoids via the portal vein, or in extravascular compartments when infection has passed the microvascular endothelium. In mammals, endotoxin sensing is mediated by circulating LPS-binding protein (LBP) which transfers LPS to a signaling receptor complex composed of a glycosylphosphatidylinositolanchored membrane-associated form of CD14 (mCD14), MD-2, and Toll-like receptor-4 (TLR4) on myeloid cells or by LBP and a soluble form of CD14 (sCD14) which transfers LPS to a MD-2/TLR4 receptor complex on nonmyeloid cells. The soluble form of CD14 is either shedded from cells bearing $\mathrm{mCD} 14$ or from direct secretion. Since sCD14 is also able to compete with $\mathrm{mCD} 14$ for LPS, a dual role for $\mathrm{SCD} 14$ has been proposed, namely an inhibitory function on systemic responses by 
promoting LPS efflux from mCD14 and transferring it to plasma lipoproteins and a stimulatory function on local inflammation in tissues that may increase resistance to bacteria [1]. Apart from that, LBP, CD14, and MD-2 have been understood to function as upstream accessory molecules that mediate physical ligand presentation, i.e., the extraction of endotoxin monomers from the bacterial outer membrane, and determine the amplitude of TLR4 activation (reviewed in [2]).

Aside from genetic variations within the TLR4 gene which have been shown to be associated with individual endotoxin sensitivity [3, 4], many lines of evidence suggest that also genetic variations within the CD14 gene do determine the outcome of LPS exposure. For instance, healthy individuals with the TT genotype at the variant position rs 2569190 have higher serum baseline levels of sCD14 than those with the CC genotype [5], TT homozygous children who were skin test positive to local aeroallergens have significantly lower total IgE levels when compared to $\mathrm{C}$ allele carriers [6], and in Finish children, a gene-environment interaction between $\mathrm{T}$ allele carriers and Helicobacter pylori antibodies on serum total $\operatorname{IgE}$ was shown [7]. The same polymorphism has been shown to be associated with alcoholic or cryptogenic chronic liver disease progression [8-11]; it is supposed that the T allele confers susceptibility to endogenous gut-derived LPS via an enhanced expression of CD14 on liver cells thus promoting progression of a chronic liver disease. By applying electrophoretic mobility shift assays (EMSA), this SNP has been found to lie within a GC box that contains a binding site for $\mathrm{Sp} 1, \mathrm{Sp} 2$, and $\mathrm{Sp} 3$ transcription factors. A DNA element containing the $\mathrm{T}$ allele was found to favor transcription factor binding. Moreover, luciferase reporter gene assays revealed the T allele DNA element to promote gene transcription in monocytic cells via an enhanced affinity to Sp1 and Sp2 [6, 12].

This study aimed at investigating of whether the variant position rs2569190 confers allele-specific gene expression also in a natural genomic context, i.e., whether it can be regarded as a regulatory polymorphism. The majority of SNPs which influence illness are assumed to be regulatory (rSNP) meaning that they lie outside the amino acid coding regions of genes and modify the expression level of transcripts [13]. By taking further SNPs within the CD14 gene and nearby regions with relevant minor allele frequencies (MAF; >0.1) into account, two parameters of gene transcription were analyzed in freshly prepared human peripheral blood mononuclear cells (PBMC). First, haplotype-specific chromatin immunoprecipitation (HaploChIP) using antibodies directed against serine 5 phosphorylated RNA polymerase II was applied to assess transcription initiation [14, 15]. Second, allele-specific transcript quantification (ASTQ) was applied to quantify the relative numbers of transcripts that have been generated from the two allelic gene variants. Both techniques circumvent intrinsic errors in interindividual comparisons, since comparison of alleles is made within rather than between samples [16]. Nonetheless, total numbers of CD14 transcripts were also assessed with regard to the blood donors' geno- and haplogenotypes.

\section{Materials and methods}

\section{Blood donors}

Samples of $30 \mathrm{ml}$ venous blood were taken from 42 healthy Caucasian blood donors (20 female, 22 male; mean age 31.8 years, range 23-57) who gave a written informed consent. The study was approved by the local ethical committee and conformed to the ethical principles of the 2000 Declaration of Helsinki.

\section{HaploChIP [17]}

Chromatin immunoprecipitation was conducted using the ChIP-IT Express kit (Active Motif, Rixensart, Belgium) according to the supplier's protocol. In brief, $1-10 \times 10^{6}$ cells were cross-linked using $0.37 \%$ formaldehyde for $6 \mathrm{~min}$ at room temperature. Fixation reactions were stopped by adding glycine. Cells were washed once in ice-cold PBS, resuspended in ice-cold lysis buffer, and subjected to dounce homogenization to release the nuclei. Pelleted nuclei were resuspended in digestion buffer and incubated in an enzymatic shearing cocktail for $10 \mathrm{~min}$ at $37^{\circ} \mathrm{C}$. After centrifugation, sheared chromatin was stored in aliquots at $-80^{\circ} \mathrm{C}$ until further use for immunoprecipitation or as control "input" DNA.

Chromatin corresponding to $1 \times 10^{6}$ cells was incubated with an antibody directed against serine 5 phosphorylated RNA polymerase II or with a control mock (IgG) antibody, and antibody-bound protein/DNA complexes were precipitated through use of magnetic protein G-coated beads. The captured chromatin was then subjected to reverse crosslinking and protease $\mathrm{K}$ digestion and taken up in a final volume of $100 \mu \mathrm{l}$.

Input DNA $(0.8 \mu \mathrm{l})$ and antibody captured DNA $(8 \mu \mathrm{l})$ were amplified by polymerase chain reaction (PCR) in $100 \mu \mathrm{l}$ reactions using the primers rs 2569190 for $5^{\prime}$-GAC ACA GAA CCC TAG ATG CCC T-3' and rs $2569190 \mathrm{rev}$ 5'-GTG AAC TCT TCG GCT GCC TC-3'. Cycling conditions were a 2 -min denaturation at $94^{\circ} \mathrm{C}, 28$ cycles of $30 \mathrm{~s}$ denaturation at $94^{\circ} \mathrm{C}$, and $1 \mathrm{~min}$ annealing and elongation at $60^{\circ} \mathrm{C}$ followed by a final elongation step for $7 \mathrm{~min}$ at $60^{\circ} \mathrm{C}$. PCR products were subjected to restriction fragment length polymorphism (RFLP) analysis. The 
products (201 bp) were digested with HaeIII (Fermentas, St. Leon-Rot, Germany) overnight. Samples were analyzed in the Agilent 2100 bioanalyzer using the DNA 1000 LabChip kit (Agilent Technologies, Böblingen, Germany) according to the supplier's instructions.

Preparation of PBMC and isolation of total cellular RNA

PBMC from approximately $30 \mathrm{ml}$ of heparinized peripheral blood were prepared by Ficoll density centrifugation using guanidinium isothiocyanate, and total cellular RNA was isolated by $\mathrm{CsCl}$ density gradient ultracentrifugation essentially as described [18].

\section{ASTQ}

Discrimination of transcripts using rs4914 as a marker SNP was achieved by two different read-out assays. First, discrimination and quantification of transcript variants were achieved by real-time PCR using two differently fluorescence dye-labeled allele-specific minor groove binder (MGB) probes for the SNP rs4914 (VIC 5'-CCT TGG AGE AGC AC-3', FAM 5'-CTT GGA GCAA GCA CC-3') and gene-specific primers (rs4914 for $5^{\prime}$-CTG AAG CCA AGG CAG TTT GAG T-3', rs4914 rev 5'-TCC CAG CCT GTG CAC GTT-3'). The assay was validated to be allelespecific (absence of a signal from the second probe in homozygote genomic DNA (gDNA) samples) and quantitative as judged from reaction efficiencies and from comparable signals in heterozygote gDNA samples (data not shown).

Secondly, discrimination and quantification of transcript variants was carried out by RFLP analyses: A product of $201 \mathrm{bp}$ in size was generated by conventional PCR (CD14 exon2 for 5'-GCC CGA GGT GGA TAA CCT G-3', CD14 exon2 rev 5'-GTC CAT TCA TTA TTC TGT CTT GGA TC-3'), purified and concentrated using the PrepEase gel extraction kit (USB, Cleveland, OH, USA), and subjected to digestion by SatI and Alw21I (Fermentas) for $2 \mathrm{~h}$. SatI cleaves $\mathrm{C}$ allele products into fragments of 55 and $146 \mathrm{bp}$ in size, whereas digestion by Alw21I leads to fragments of 106 and $95 \mathrm{bp}$ in size for $\mathrm{C}$ allele products and to fragments of 106,50 , and $40 \mathrm{bp}$ in size for $\mathrm{G}$ allele amplicons, respectively. Samples were separated and analyzed in the Agilent 2100 bioanalyzer using the DNA 1000 LabChip kit (Agilent Technologies). The relative amount of $\mathrm{C}$ and $\mathrm{G}$ allele products and of hybrids was calculated.

\section{Sequencing}

Three overlapping regions of the CD14 gene and nearby regions spanning 2,101 nucleotides (Fig. 1) were amplified using gDNA as template, GoTaq Colorless Master Mix
(Promega GmbH, Mannheim, Germany), and $0.36 \mu \mathrm{M}$ of primers each (for1 5'-GCC GAG ATC ATG GCA-3', rev1 5'-ACC CTG ATC ACC TCC CCA C-3'; for 2 5'-CCC CTC CCT GAA ACA TCC TT-3', rev2 5'-GAG TGT GCT TGG GCA ATG CT-3'; for3 5'-GGA CTT GCA CTT TCC AGC TTG-3', rev3 5'-GCA CAT AGC AGA CAT CCA ATA AAG G-3'). Products were purified following the spin protocol of the QIAquick PCR Purification Kit (Qiagen, Hilden, Germany) and sequenced using an automated sequencer (SeqLab, Göttingen, Germany). Alignments and analyses were done using the Basic Local Alignment Search Tool program of National Center for Biotechnology Information (NCBI; National Institutes of Health, Bethesda, MD, USA) and version 2.01 of the software Chromas LITE (Technelysium Pty Ltd, Tewantin, QLD, Australia).

\section{Genotyping}

Genomic DNA was purified from whole blood samples using the QIAamp DNA Blood Mini Kit following the blood and body fluid spin protocol (Qiagen). Typically, genotype determinations were performed by using $10 \mathrm{ng}$ of PBMC-derived gDNA.

Genotyping of the variant position of interest, rs2569190, was carried out by allelic discrimination in 5'-nuclease realtime PCR assays in the sequence detection system ABI prism 7000 (Applied Biosystems, Darmstadt, Germany) according to the supplier's instructions. In brief, gDNA was amplified with $0.36 \mu \mathrm{M}$ of primers each (forward 5'-CTA GAT GCC CTG CAG AAT CCT T-3', reverse 5'-CCC TTC CTT TCC TGG AAA TAT TGC A-3') using the TaqMan Universal Master Mix (Applied Biosystems). Allelic discrimination was achieved by adding $0.2 \mu \mathrm{M}$ differentially fluorescence dyelabeled allele-specific MGB probes (rs2569190: VIC 5'-CCT GTT ACG GTC CCC CT-3', FAM 5'-CTG TTA CGG $\underline{\text { CCC }}$ CCC T-3').

Genotyping of the variant position rs4914, the marker SNP for ASTQ, was performed in the sequence detection system ABI prism 7000 by using a commercially available TaqMan genotyping assay (C_8724832_10) according to the supplier's instructions. Discrimination of genomic DNA and transcript variants at SNP rs705 within the imprinted gene small nuclear ribonucleoprotein polypeptide $\mathrm{N}$ (SNRPN) was carried out using a commercially available genotyping assay from ABI (C_2066555_10).

Genotyping of the SNPs rs5744455 and rs2563298 was performed by tetra-primer amplification refractory mutation system (ARMS) PCR as introduced by Ye et al. [19]. In brief, gDNA was used as template in conventional amplification reactions using $0.18 \mu \mathrm{M}$ of each primer (primer rs574 tetra $\mathrm{T}$ for $5^{\prime}$ AAG GAA GGG GGA ATT TTT CTT TAG CCT $3^{\prime}$, primer rs574 tetra C rev 5' GGT AGA ATT AGG TTC AAG AAA AGG AAG GTG 3', 


\section{a}

CD14 [minus strand]

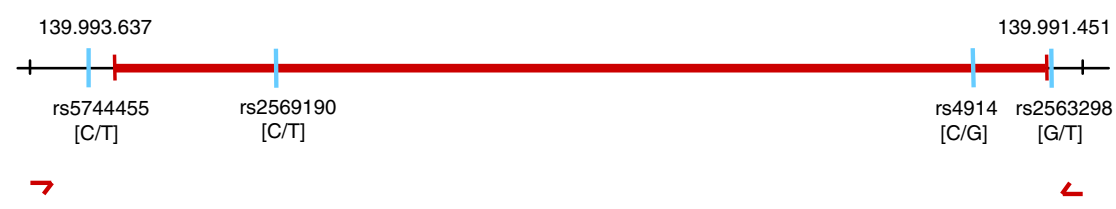

gene

bases / contig position human chrom 5

SNP \#

flanking sequencing primer

\section{b}

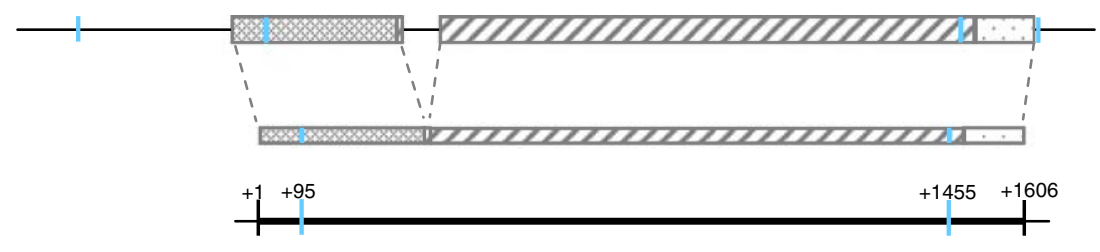

exon / intron structure of variant 1

transcript variant 1 (NM_000591.2)

mRNA position with regard to transcription start site

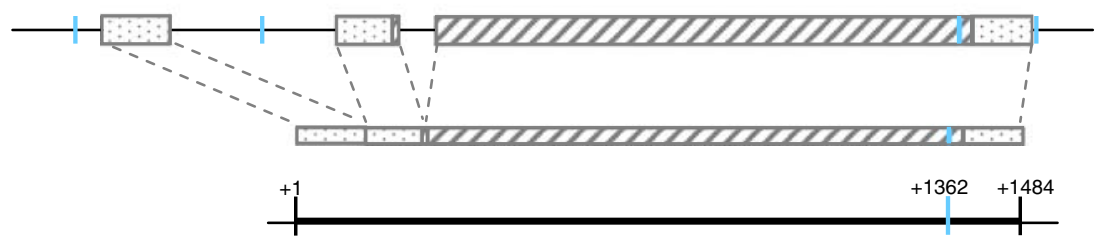

exon / intron structure of variant 2

transcript variant 2 (NM_001040021.1)

mRNA position with regard to

transcription start site

Fig. 1 Schematic presentation of the structure of the CD14 gene and of the two transcript variants. a In humans, the CD14 gene is located on chromosome 5 . The gene and the nearby $5^{\prime}$ and $3^{\prime}$ regions contain four SNPs with MAFs $>0.1$ as indicated. Presence of these four SNPs was confirmed by sequencing the indicated region. b Differential

primer rs574 tetra for 5' CTG AGC AAC AGA GCA AGA CTC TAT CTC A 3', primer rs574 tetra rev 5' CAT TTC TTT GAC TTC TTC CTT GTC TTG G 3' for rs5744455, and primer rs 2563 tetra $\mathrm{G}$ for $5^{\prime} \mathrm{CCC}$ ACC TTT ATT AAA ATC TTA AAG AAC AGG 3', primer rs2563 tetra T rev 5' TAA AGG TCT GTT AAA TGA ATG ACA CGA AA $3^{\prime}$, primer rs 2563 tetra for $5^{\prime}$ TTT GCC TAA GAT CCA AGA CAG AAT AAT G 3', and primer rs 2563 tetra rev 5' TTT CTT AGG GAG TTA GGA TGA AGA AAG C 3' for rs 2563298). Amplicons were separated in 4\% agarose gels. Product patterns of 277/142, 277/142/192, and 277/192 bp correspond to CC, CT, and TT genotypes of rs5744455 and patterns of $247 / 143,247 / 143 / 163$, and $247 / 163$ bp to GG, GT, and TT rs2563298 genotypes, respectively.

\section{Haplotyping}

Haplotyping of the four respective SNPs was performed by a combination of ARMS-PCR and genotyping assays. Amplicons spanning the four SNPs of interest were generated using rs5744455 and rs2569190 allele-specific splicing results in the existence of two transcript variants. Both of them do contain rs4914, the marker SNP used for ASTQ. In transcript variant 1, rs 2569190 is located within the $5^{\prime}$ UTR; because of its intronic location in variant 2, rs 2569190 is absent from transcript variant 2

primers (rs574 tetra $\mathrm{T}$ for, rs574 tetra $\mathrm{C}$ for $5^{\prime}$-AAG GAA GGG GGA ATT TTT CTT TAG CCC-3', CD14 tetra3-rev 5'-TTT TCT TGA GGA GGA CAG ATA GGG TTT C-3', and rs2569190 C for 5'-CCT GCA GAA TCC TTC CTG TTA CTG C-3', rs2569190 T for 5'-CCC TGC AGA ATC CTT CCT GTT ACT GT-3', CD14 tetra3-rev, respectively) in a conventional $25-\mu$ l PCR with an extended elongation step $\left(2.5 \mathrm{~min} 72^{\circ} \mathrm{C}\right)$ in a FlexCycler (Analytik Jena, Jena, Germany). The amplicons were then subjected either to $5^{\prime}$-nuclease genotyping assays for rs 2569190 or rs4914 (see above) or to allele-specific amplification using tetra-primers for rs2563298 (rs2563 tetra $\mathrm{G}$ for, rs2563 tetra $\mathrm{T}$ rev, rs2563 tetra for, rs2563 tetra rev) in standard SYBR green reactions.

\section{Quantification of CD14 mRNA}

Quantification of gene expression was performed by realtime reverse transcriptase (RT)-PCR using the sequence detection system ABI prism 7000 (Applied Biosystems) as described [18]. Commercially available TaqMan gene 
expression assays were used for determination of CD14 (Hs00169122g1), CD11b (Hs00355885m1), and GAPDH (Hs99999905m1) transcripts. To discriminate between transcript variant 1 and 2, the TaqMan genotyping assays for the SNPs rs2569190 and rs4914 were used in parallel.

\section{Results}

Analysis of allele-specific transcriptional activity in situ within a natural genomic context can be performed (a) on the level of gDNA determining serine 5 phosphorylated RNA polymerase II loading by HaploChIP technology as a parameter of transcription initiation and (b) on the level of mRNA determining the relative number of transcripts originating from two gene copies by ASTQ. Both techniques do not rely on the comparison of individuals but can be carried out with material from single individuals who are heterozygous at the position of interest. Whereas material from individuals who are heterozygous at a respective position is the only requirement for HaploChIP analyses, ASTQ requires material from individuals who are additionally heterozygous at a second SNP within the transcript (if the SNP of interest is located, e.g., in a nontranscribed regulatory region) and which must be used to discriminate between transcripts derived from one or the other sister chromosome. According to NCBI database, the CD14 transcript does contain a SNP suitable for ASTQ analysis, rs4914, due to its location (Fig. 1) and due to a sufficiently high MAF that should allow identification of double heterozygous individuals. Accordingly, from a total of 42 healthy individuals who were genotyped at positions rs2569190 and rs4914 (Table 1), 27 were found to be heterozygous at rs2569190, and seven were found to be heterozygous at both positions.

To cover all relevant SNPs within the CD14 gene and the nearby $5^{\prime}$ and $3^{\prime}$ regions, e.g., with a MAF $>0.1$, which might affect transcriptional activity, too, a panel of ten samples was sequenced spanning a region of 2,101 nucleotides (see Fig. 1a). Alignment of the sequences confirmed the four known SNPs rs5744455, rs2569190, rs4914, and rs2563298 and the absence of any further with relevant MAFs (data not shown). Genotyping of rs5744455 and rs2563298 (Table 1) and haplotyping of the four respective loci was performed by a combination of ARMSPCR and 5'-nuclease genotyping and revealed a total of five haplotypes (Table 2) resulting in a total of eleven haplogenotypes. C-T-C-G, the most frequent haplotype, was also the only one carrying a $\mathrm{T}$ allele at the position of interest, rs2569190.

HaploChIP analysis was performed on freshly prepared PBMC from five healthy rs2569190 heterozygous blood donors. Sheared chromatin from formalin-fixed cells served as the "input" control. Input chromatin and chromatin that was captured by an antibody directed against serine 5 phosphorylated RNA polymerase II, the "output" DNA, were analyzed for the ratio of fragments carrying the $\mathrm{C}$ or the $\mathrm{T}$ allele at position $\mathrm{rs} 2569190$ by RFLP analysis. Whereas input chromatin was found to contain about the same numbers of $\mathrm{C}$ and $\mathrm{T}$ variant fragments, the chromatin of all volunteers that had been captured by anti-RNA polymerase II antibodies was found to contain about twice the numbers of $\mathrm{T}$ variant fragments (Fig. 2a) suggesting that RNA polymerase II preferentially binds fragments containing the rs $2569190 \mathrm{~T}$ allele. As an additional control, chromatin was also captured by a mock isotype (IgG) antibody. The mock captured material was found to contain nearly same amounts of $\mathrm{C}$ and $\mathrm{T}$ allele fragments $(\mathrm{T} / \mathrm{C}$ ratios of $1.18,1.02$, and 1.21$)$ as with the input control $(\mathrm{T} / \mathrm{C}$ ratios $1.08,1.10$, and 1.11 , respectively), while the output material was found to contain more $\mathrm{T}$ allele fragments $(\mathrm{T} / \mathrm{C}$ ratios $1.90,1.65$, and 1.80 , respectively; three independent samples, data not shown). An analysis of HaploChIP data with regard to haplogenotypes revealed the haplotype C-T$\mathrm{C}-\mathrm{G}$ that one to be preferentially captured by anti-RNA polymerase II antibodies, irrespectively of its genomic counterpart (Fig. 2b). As an additional control for validation of HaploChIP results, input and output chromatins were analyzed for the presence of two alleles of the SNP rs705 located within the imprinted gene SNRPN. Whereas input DNA contained similar amounts of each allele, immunoprecipitated material captured by antibodies directed against serine 5 phosphorylated RNA polymerase II contained only one allele as expected for the monoallelic expression (see Fig. 3b, lower panel).
Table 1 Genotype distribution
among healthy blood donors

Table 1 Genotype distributio
among healthy blood donors

\footnotetext{
${ }^{\text {a }}$ [wild-type (wt), variant (v)]

${ }^{\mathrm{b}}$ Exact test for Hardy-Weinberg equilibrium
}

${ }^{\mathrm{a}}$ [wild-type (wt), variant (v)]
${ }^{\mathrm{b}}$ Exact test for Hardy-Weinberg
equilibrium

\begin{tabular}{llllll}
\hline \multicolumn{7}{l}{ Genotype distribution } & & \\
\cline { 2 - 6 } & wt/wt & wt/v & v/v & MAF & $P^{\mathrm{b}}$ \\
\hline $\operatorname{rs} 5744455[\mathrm{C} / \mathrm{T}]^{\mathrm{a}}$ & $22(52.4 \%)$ & $18(42.9 \%)$ & $2(4.7 \%)$ & 0.26 & 0.7 \\
$\operatorname{rs} 2569190[\mathrm{C} / \mathrm{T}]^{\mathrm{a}}$ & $10(23.8 \%)$ & $27(64.3 \%)$ & $5(11.9 \%)$ & 0.44 & 0.07 \\
$\mathrm{rs} 4914[\mathrm{C} / \mathrm{G}]^{\mathrm{a}}$ & $31(73.8 \%)$ & $9(21.4 \%)$ & $2(4.8 \%)$ & 0.15 & 0.23 \\
$\mathrm{rs} 2563298[\mathrm{G} / \mathrm{T}]^{\mathrm{a}}$ & $22(52.4 \%)$ & $17(40.5 \%)$ & $3(7.1 \%)$ & 0.27 & 1 \\
\hline
\end{tabular}


Table 2 Haplotype distribution among the healthy individuals under study

$\begin{array}{lrr} & \text { C-T-C-G } & 37 \\ & \text { T-C-C-G } & 22 \\ \text { C-C-G-T } & 13 \\ & \text { C-C-C-T } & 10 \\ \text { a rs5744455-rs2569190- } & \text { C-C-C-G } & 2 \\ \text { rs4914-rs2563298 } & \end{array}$

To investigate whether a CD14 gene with a $\mathrm{T}$ allele at position rs 2569190 is more actively transcribed than the $\mathrm{C}$ allele counterpart, ASTQ was performed on cDNA samples from double heterozygous donors using rs 4914 for discrimination of transcripts. Discrimination of transcripts was achieved by two independent read-out assays: by RFLP analyses and by $5^{\prime}$-nuclease assays using differently labeled allele-specific probes. The amounts of $\mathrm{C}$ and $\mathrm{G}$ allelic transcript variants were not found to be different in all samples studied as they were neither, expectedly, within two samples from donors who are homozygous for the $\mathrm{C}$ allele at rs 2569190. These results were achieved irrespectively of the technique applied (Fig. 3). Accordingly, analysis of ASTQ data with regard to haplogenotypes revealed that the number of transcripts originating from $\mathrm{C}$ T-C-G haplotypes does not exceed the number originating from the other haplotypes (Fig. 3a). As a control for allelic discrimination via RFLP analyses, cDNA samples from two homozygotes were mixed at various ratios and analyses yield expected results (Fig. 3a). As a control for allelic discrimination via allele-specific probes, gDNA and cDNA samples from individuals who were heterozygous at SNP rs705 within the imprinted gene SNRPN were analyzed for the ratio of the two alleles $G$ and $A$. While in gDNA samples both alleles were detectable at nearly same amounts, corresponding cDNA samples contained either one or the other (Fig. 3b, lower panel).

The existence of two different CD14 mRNA variants, one of which carrying the SNP of interest within its $5^{\prime} \mathrm{UTR}$ (variant 1) and the other not at all (variant 2; Fig. 1b), might bias quantification of allelic transcript expression, e.g., by different half life times. To determine the relative proportion of transcript variants 1 and 2 within the cDNA samples, cDNA samples were analyzed for the total amount of CD14 transcripts on the one hand and for the amount of transcript variant 1 by using an assay spanning rs 2569190 . Whereas the number of transcripts containing rs4914 equals
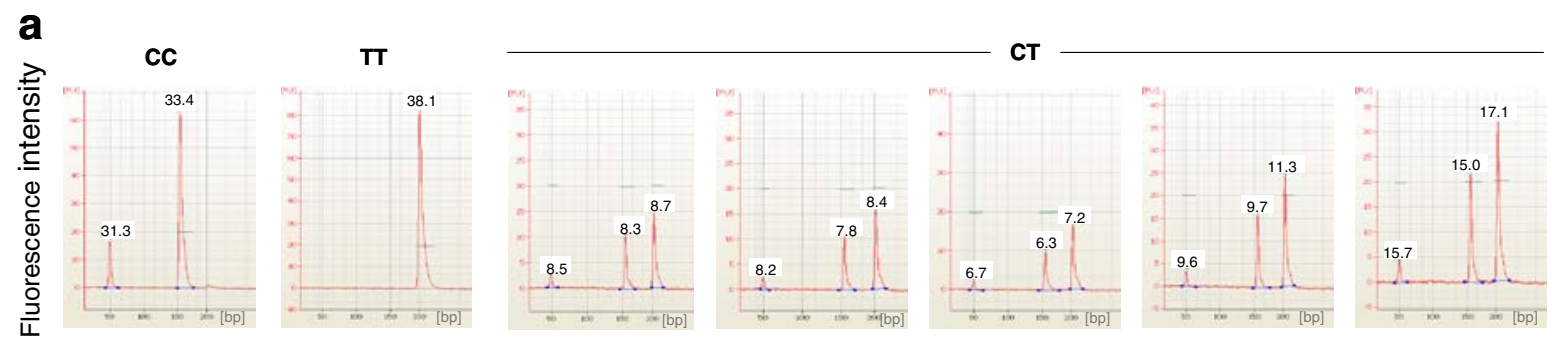

Input
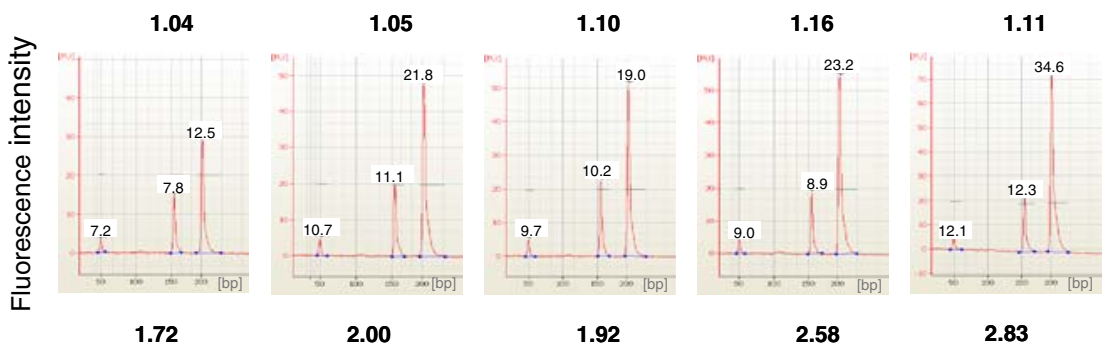

T/C ratio

b
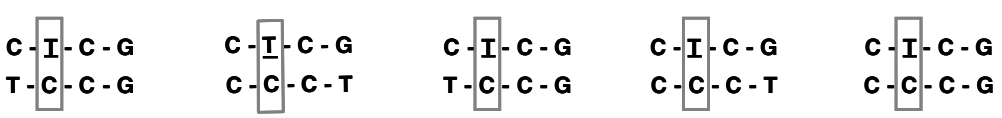

Fig. 2 Chromatin immunoprecipitation. a Formalin-fixed and enzymatically sheared chromatin from five healthy individuals heterozygous at rs2569190 was immunoprecipitated with antibodies directed against RNA polymerase II as described in the "Materials and methods" section or was left untreated as an "input" control. Purified input and output DNA samples were subjected to amplification spanning rs2569190. Products of $201 \mathrm{bp}$ in size were subjected to restriction enzyme digestion with HaeIII. Restriction digest analysis was carried out in a bioanalyzer yielding molar amounts of each fragment as given. Undigested fragments represent DNA of the T allele. Fragments of 49 and $152 \mathrm{bp}$ in size represent DNA of the C allele. Material from homozygous individuals (TT and CC) is shown as a control for complete and specific enzymatic activity. b Haplotypes (rs5744455-rs2569190-rs4914-rs2563298) of the five donors are given 
a

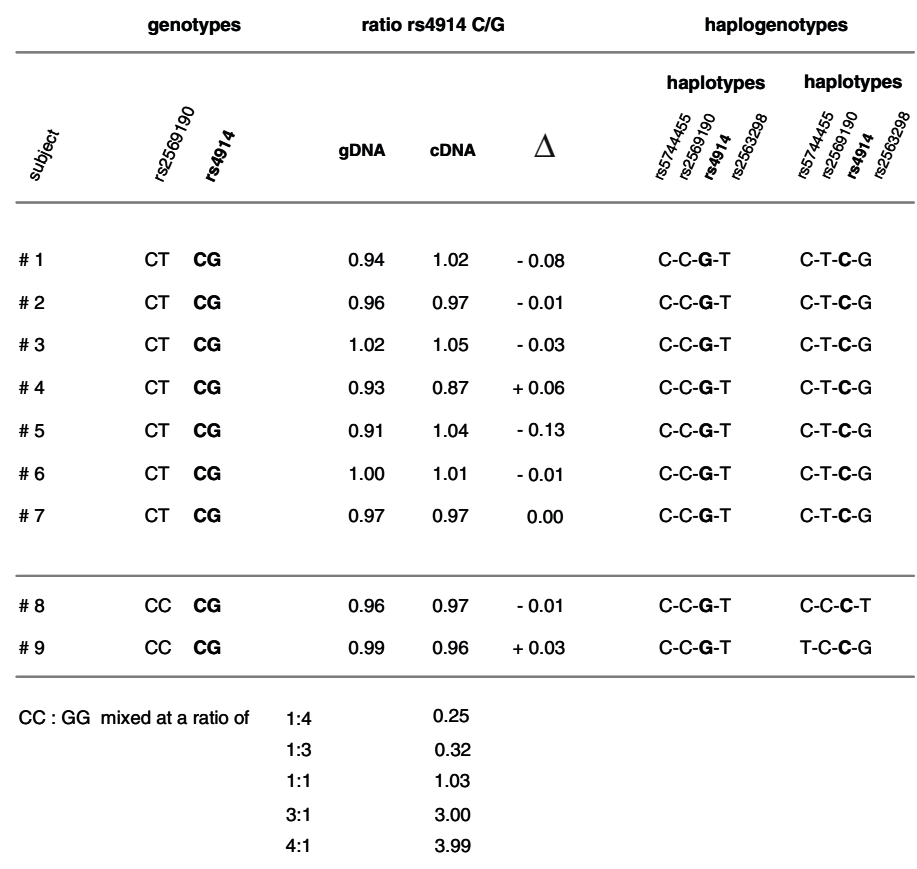

b
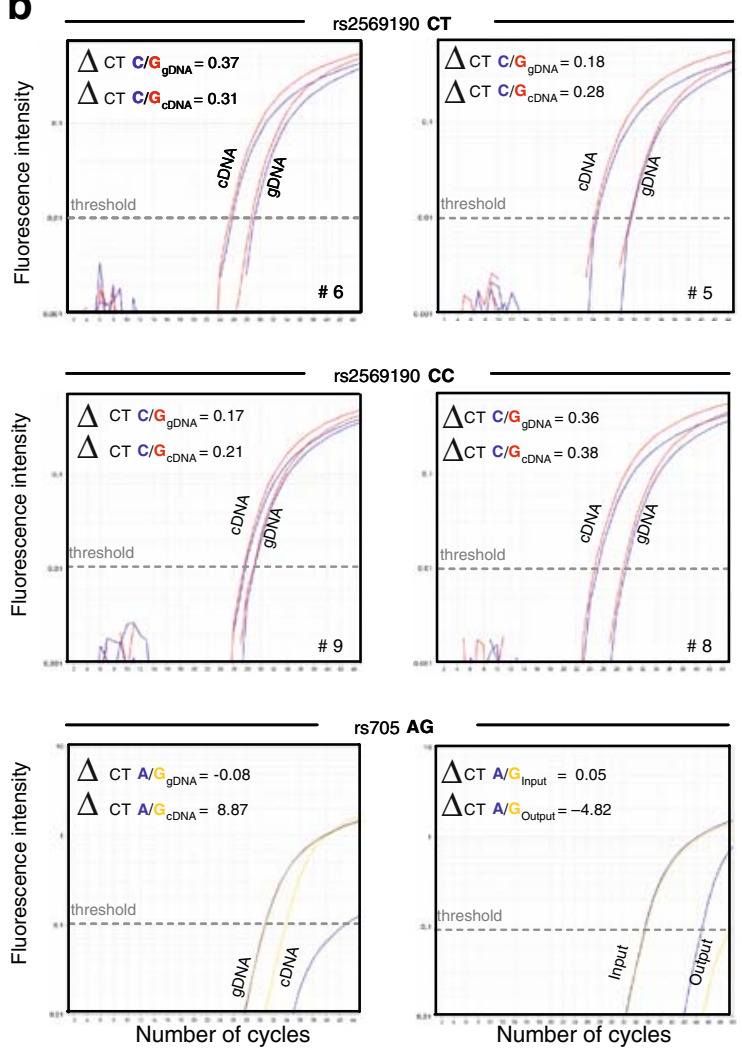

Fig. 3 ASTQ analyses. The relative amount of mRNA species carrying the $\mathrm{C}$ or the $\mathrm{G}$ allele at position rs4914 isolated from freshly prepared human PBMC samples was analyzed by RFLP and by 5'nuclease assays using allele-specific probes. a RFLP data for all seven double heterozygous individuals are given as the ratio of rs $4914 \mathrm{C} / \mathrm{G}$ alleles. Samples for two individuals homozygous at position rs2569190 (\#8 and \#9) as well as corresponding gDNA samples served as controls. cDNA samples that had been adjusted for the amount of CD14 mRNA by quantitative gene expression assays were

the number of total CD14 mRNA, the number of nucleic acids containing rs 2569190 was about 100 -fold lower and in the range of residual gDNA (Fig. 4). PBMC thus were found to contain nearly exclusively transcript splice variant 2. Analysis of total CD14 mRNA from freshly isolated PBMC failed to reveal a significant relationship with either the four single SNPs and with haplogenotypes when blood donors are divided into groups with none, one, or two C-TC-G haplotypes (Fig. 5).

\section{Discussion}

According to earlier knowledge, the SNP rs2569190 was supposed to be located at position -159 within the promoter of the CD14 gene. On the basis of association studies, reporter gene assays, and EMSAs, this SNP was suggested to affect transcriptional activity by a differential binding of Sp1, Sp2, and Sp3 transcription factors [6, 8-10, 12]. mixed at various ratios yielding expected $\mathrm{C} / \mathrm{G}$ ratios. Haplogenotypes are indicated. b 5'-Nuclease assays were performed in triplicates. Data from one representative determination are given for two representative samples (\#5 and \#6; top), for two homozygous controls (\#8 and \#9; middle), and for a control of the imprinted gene SNPRN for genomic and cDNA and for HaploChIP material (bottom). $\triangle \mathrm{CT}$ values for the rs4914 C (blue) and G alleles (red) in cDNA samples were not significantly different from the respective gDNA control

Intriguingly, the impact of this SNP was shown to be different in monocytes and hepatoma cells, most probably due to a cell type specific different transcription factor pattern [12]. According to more recent knowledge, rs2569190 is located downstream of the transcription start site, and two splice variants do exist (Fig. 1). Its relative position within the (im-) mature transcripts depends on the splice variant; while rs2569190 is located within the 5' UTR of the transcript variant 1 , this SNP is lacking in transcript variant 2 due to its intronic position.

PBMC were found to contain variant 2 CD14 transcripts (Fig. 4). Because of the absence of that SNP within the transcript, an effect of rs2569190 on transcript stability in PBMC can be ruled out. On the DNA level, the SNP might affect transcription factor binding as it is located in a transcription factor binding motif, a GC box, known to bind transcription factors $\mathrm{Sp} 1, \mathrm{Sp} 2$, and $\mathrm{Sp} 3$. LeVan et al. have shown both in EMSAs and in reporter gene assays that the $\mathrm{T}$ allele confers higher transcriptional activity in a mono- 


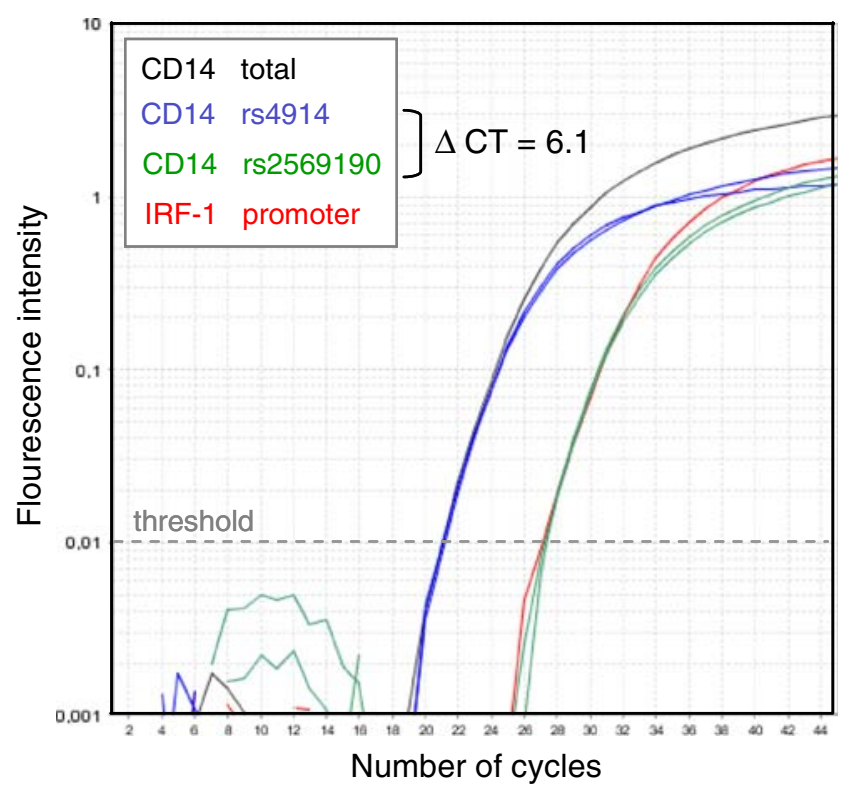

Fig. 4 Relative proportions of CD14 splice variants within PBMC cDNA samples. cDNA samples from seven double heterozygous individuals were analyzed for the amount of nucleic acids detected by an assay specific for total CD14 mRNA, by an assay specific for a region spanning rs4914, by an assay specific for a region spanning rs 2569190 , and by an assay specific for gDNA (IRF-1 promoter). The amount of products containing rs4914 equals the amount of total CD14 mRNA, whereas the amount of products containing rs 2569190 equals that of gDNA

cytic cell line by preferential binding of the activating Sp1 and Sp2 transcription factors [12].

For the in vivo situation, i.e., in a natural genomic context, we found a stronger recruitment of RNA polymerase II to T allele variants. Transcription is known to follow a complex phosphorylation/dephosphorylation cycle in which RNA polymerase II is underlying a recycling process [15]. In brief, after assembly onto promoter sequences with general transcription factors (preinitiation), the RNA polymerase II core enzyme is phosphorylated on serines at position 5 by CDK7. Later on, after initiation of transcription, elongation requires phosphorylation on serines at position 2 by CDK9, a subunit of a transcription elongation factor. Polyadenylation signals transcription to terminate, to relieve RNA polymerase

Fig. 5 CD14 mRNA expression in healthy donors with regard to their (haplo-)genotypes at four variant positions. Total CD14 mRNA was quantified in PBMC samples from 42 healthy blood donors using a commercially available gene expression assay by real time RT-PCR. Data were analyzed with regard to genotypes at the four respective positions and with regard to haplogenotypes as indicated. CD14 mRNA expression was not found to be different when donors with different (haplo-)genotypes were compared to each other, irrespectively of whether data were related to GAPDH as a reference transcript as shown or whether they were related to $\mathrm{CD} 11 \mathrm{~b}$ as a reference. Medians are given; for rs5744455 and rs4914, medians refer to wildtype homozygotes and variant allele carriers, respectively
II off its template, and finally to be dephosphorylated. HaploChIP analyses were performed using an antibody directed against serine 5 phosphorylated RNA polymerase II. Our data thus indicate that in the in vivo situation, the T allele confers stronger transcription initiation than the wildtype allele. Given that transcription factor binding might
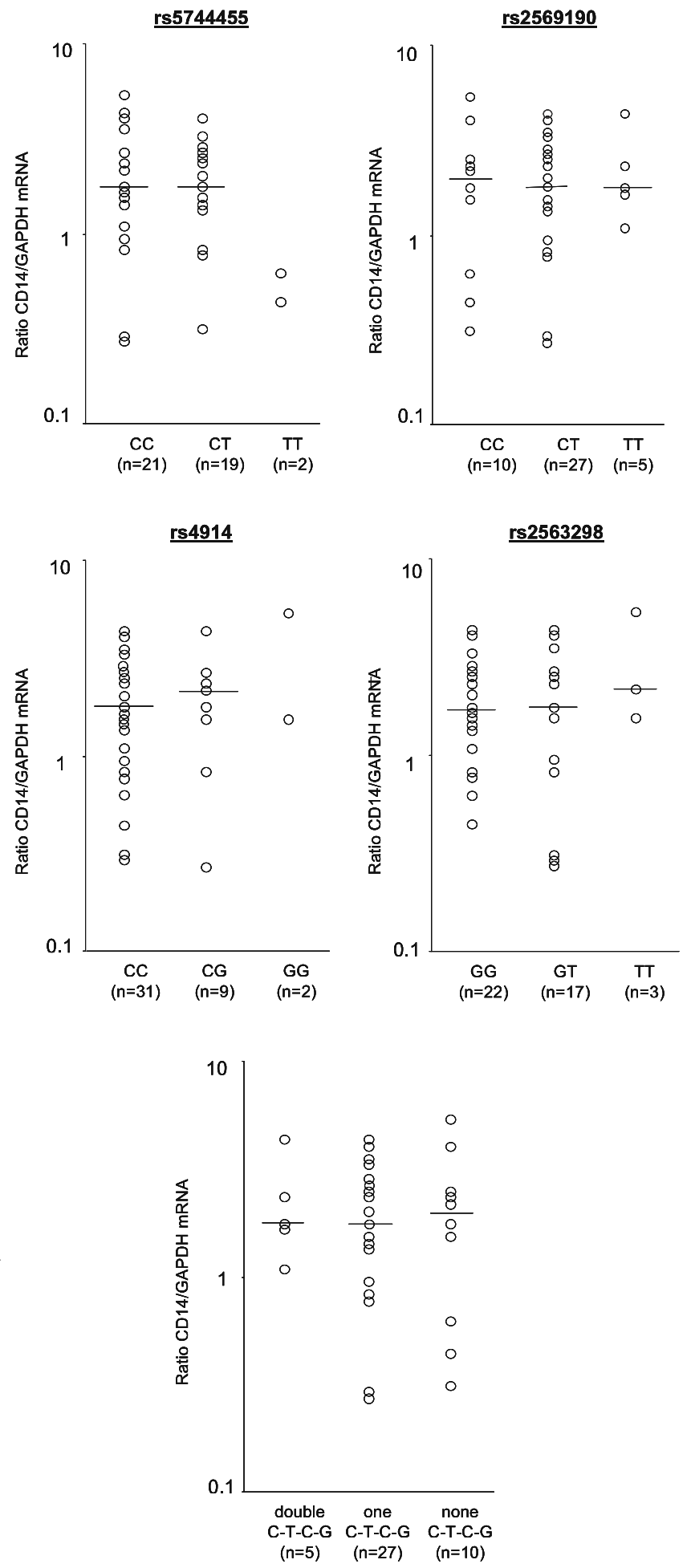
stabilize RNA polymerase II binding, our data are thus in line with the findings by LeVan et al. [12].

By taking the number of transcripts as an end-point of transcription and by using two independent techniques for allelic discrimination, however, we found similar amounts of allelic mRNA variants. This finding is in accordance with data on total CD14 transcripts which do not differ in PBMC from donors with different genotypes (Fig. 5). We thus suppose that the $\mathrm{T}$ allele, or the C-T-C-G haplotype, might be advantageous for transcription initiation but not necessarily relevant for CD14 mRNA expression at least in PBMC.

While the rs2569190 $\mathrm{T}$ allele gene copy has been demonstrated to be preferentially loaded by RNA polymerase II in PBMC, endotoxin sensitivity appears not to be related to allele-specific CD14 gene expression in PBMC. This finding is in line with others showing different effects of regulatory variants in different tissues (reviewed in [16]). As outlined in the introduction, endotoxin sensing is not only mediated by mCD14 expressing myeloid cells but also by sCD14 able to transfer LPS to TLR4/MD2 expressing nonmyeloid cells lacking mCD14. Moreover, circulating sCD14 is known to inhibit systemic inflammatory responses to LPS via its ability to remove LPS from mCD14 and to transfer it to plasma lipoproteins which mediate clearance [1]. Thus, the associations of rs2569190 TT genotype with higher sCD14 levels and with lower levels of IgE might reflect an effect of that SNP independent on myeloid mCD14 but dependent on nonmyeloid, e.g., hepatic, sCD14 expression or action.

As sCD14 is described to have stimulatory effects in extravascular compartments, the association of rs $2569190 \mathrm{~T}$ allele and progression in alcoholic liver disease might be also due to a better sensing of (endogenous) LPS from the gut via sCD14-mediated transfer of LPS to nonmyeloid liver cells. Based on the observation that hepatic stellate cells express TLR4 and respond to its stimulation, it has been suggested that TLR-mediated direct stellate cell activation is a major pathway to induce liver fibrosis $[20,21]$.

Several lines of evidence indicate that hepatocytes are the source of sCD14 [22-24]. To address the impact of rs 2569190 on hepatic transcriptional activity of the CD14 gene, ASTQ analyses in liver tissue samples, e.g., from patients with a diagnosis of absence of liver disease, are required.

Acknowledgments The authors thank Waltraut Kopp for expert technical assistance. This work was supported by the Deutsche Forschungsgemeinschaft [grant number MI 474/1-1]. R.B. was supported by a research scholarship by the German Academic Exchange Service (DAAD). E.A. is grateful for her scholarship from Damascus University, Syria.

Open Access This article is distributed under the terms of the Creative Commons Attribution Noncommercial License which permits any noncommercial use, distribution, and reproduction in any medium, provided the original author(s) and source are credited.

\section{References}

1. Kitchens RL, Thompson PA (2005) Modulatory effects of sCD14 and LBP on LPS-host cell interactions. J Endotoxin Res 11:225229

2. Gioannini TL, Weiss JP (2007) Regulation of interactions of Gram-negative bacterial endotoxins with mammalian cells. Immunol Res 39:249-260

3. Arbour NC, Lorenz E, Schutte BC, Zabner J, Kline JN, Jones M, Frees K, Watt JL, Schwartz DA (2000) TLR4 mutations are associated with endotoxin hyporesponsiveness in humans. Nat Genet 25:187-191

4. El-Omar EM, Ng MT, Hold GL (2008) Polymorphisms in Tolllike receptor genes and risk of cancer. Oncogene 27:244-252

5. Levan TD, Michel O, Dentener M, Thorn J, Vertongen F, Beijer L, Martinez FD (2008) Association between CD14 polymorphisms and serum soluble CD14 levels: effect of atopy and endotoxin inhalation. J Allergy Clin Immunol 121:434-440 e431. Epub 2007 Oct 2018

6. Baldini M, Lohman IC, Halonen M, Erickson RP, Holt PG, Martinez FD (1999) A Polymorphism* in the 5' flanking region of the CD14 gene is associated with circulating soluble CD14 levels and with total serum immunoglobulin E. Am J Respir Cell Mol Biol 20:976-983

7. Virta M, Pessi T, Helminen M, Seiskari T, Kondrashova A, Knip M, Hyoty H, Hurme M (2008) Interaction between CD14-159C\&gt; T polymorphism and Helicobacter pylori is associated with serum total immunoglobulin E. Clin Exp Allergy 38:1929-1934

8. Meiler C, Muhlbauer M, Johann M, Hartmann A, Schnabl B, Wodarz N, Schmitz G, Scholmerich J, Hellerbrand C (2005) Different effects of a CD14 gene polymorphism on disease outcome in patients with alcoholic liver disease and chronic hepatitis C infection. World J Gastroenterol 11:6031-6037

9. Jarvelainen HA, Orpana A, Perola M, Savolainen VT, Karhunen PJ, Lindros KO (2001) Promoter polymorphism of the CD14 endotoxin receptor gene as a risk factor for alcoholic liver disease. Hepatology 33:1148-1153

10. Von Hahn T, Halangk J, Witt H, Neumann K, Muller T, Puhl G, Neuhaus P, Nickel R, Beuers U, Wiedenmann B, Berg T (2008) Relevance of endotoxin receptor CD14 and TLR4 gene variants in chronic liver disease. Scand J Gastroenterol 43:584-592

11. Campos J, Gonzalez-Quintela A, Quinteiro C, Gude F, Perez LF, Torre JA, Vidal C (2005) The $-159 \mathrm{C} / \mathrm{T}$ polymorphism in the promoter region of the CD14 gene is associated with advanced liver disease and higher serum levels of acute-phase proteins in heavy drinkers. Alcohol Clin Exp Res 29:1206-1213

12. LeVan TD, Bloom JW, Bailey TJ, Karp CL, Halonen M, Martinez FD, Vercelli D (2001) A common single nucleotide polymorphism in the CD14 promoter decreases the affinity of Sp protein binding and enhances transcriptional activity. J Immunol 167:5838-5844

13. Buckland PR (2006) The importance and identification of regulatory polymorphisms and their mechanisms of action. Biochim Biophys Acta 1762:17-28

14. Charles Knight J (2005) HaploChIP: an in vivo assay. Methods Mol Biol 311:49-60

15. Palancade B, Bensaude O (2003) Investigating RNA polymerase II carboxyl-terminal domain (CTD) phosphorylation. Eur J Biochem 270:3859-3870

16. Pastinen T, Hudson TJ (2004) Cis-acting regulatory variation in the human genome. Science 306:647-650

17. Knight JC, Keating BJ, Rockett KA, Kwiatkowski DP (2003) In vivo characterization of regulatory polymorphisms by allelespecific quantification of RNA polymerase loading. Nat Genet 33:469-475 Epub 2003 Mar 2010 
18. Mihm S, Frese M, Meier V, Wietzke-Braun P, Scharf JG, Bartenschlager R, Ramadori G (2004) Interferon type I gene expression in chronic hepatitis C. Lab Invest 84:1148-1159

19. Ye S, Dhillon S, Ke X, Collins AR, Day IN (2001) An efficient procedure for genotyping single nucleotide polymorphisms. Nucleic Acids Res 29:E88

20. Seki E, De Minicis S, Osterreicher $\mathrm{CH}$, Kluwe J, Osawa Y, Brenner DA, Schwabe RF (2007) TLR4 enhances TGF-beta signaling and hepatic fibrosis. Nat Med 13:1324-1332

21. Paik YH, Schwabe RF, Bataller R, Russo MP, Jobin C, Brenner DA (2003) Toll-like receptor 4 mediates inflammatory signaling by bacterial lipopolysaccharide in human hepatic stellate cells. Hepatology 37:1043-1055

22. Bas S, Gauthier BR, Spenato U, Stingelin S, Gabay C (2004) CD14 is an acute-phase protein. J Immunol 172:4470- 4479

23. Pan Z, Zhou L, Hetherington CJ, Zhang DE (2000) Hepatocytes contribute to soluble CD14 production, and CD14 expression is differentially regulated in hepatocytes and monocytes. J Biol Chem 275:36430-36435

24. Su GL, Dorko K, Strom SC, Nussler AK, Wang SC (1999) CD14 expression and production by human hepatocytes. J Hepatol $31: 435-442$ 